\title{
NECESSIDADES PSICOSSOCIAIS DE PACIENTES SUBMETIDOS À CIRURGIA DO APARELHO DIGESTIVO - UMA ASSISTEKNCIA SISTEMATIZADA 1
}

\author{
OLGA CHIZUE TAKAHASHI ${ }^{2}$
}

TAKAHASHI, O.C. Necessidades psicossociais de pacientes submetidos à cirurgia do aparelho digestivo: uma assistência sistematizada. Semina: Ci. Biol/Saúde, Londrina, v. 13, n. 2, p. 69 - 74, jun. 1992.

\section{RESUMO}

Este trabalho teve como principal objetivo, a verificaçāo do nivel de satisfaçăo das necessidades psicossociais de informaçăo, de segurança e de afeto-estima, percebidos pelos pacientes submetidos a cirurgias do aparelho digestivo que receberam assistência de enfermagem sistematizada e individualizada. Essa assistência foi implementada desde as primeiras 24 horas após a admissăo do paciente no hospital, no perfodo pré-operatório, até o $4^{9}$ dia do pós-operatónio ou anterior a essa data, quando o paciente recebia alta hospitalar. A metodologia desenvolvida favoreceu o estabelecimento de uma interaçăo positiva enfermeiropaciente, proporcionando ao paciente, oportunidade para esclarecer suas dúvidas e receber informaçōes sobre todos os prováveis acontecimentos que poderia vivenciar durante essa hospitalização para cirurgia. Pela análise dos resultados, vorificou-se que os niveis de satisfaçắo das necessidades de informação, de segurança e de afeto-estima apresentaram uma média amostraf de respectivamente 79, 95 e 89 pontos, numa escala de 0 a 100, representando níveis aceitáveis no contexto deste trabalho.

PALAVRAS-CHAVE: Assistência de enfermagem; Necessidades psicossociais; Pré-operatório; Pós-operatório; Sistematizaçăo da assistência; Informaçāo; Paciente cirúrgico; Processo de enfermagem; Avaliação; Nivel do satisfaçāo

\section{1 - INTRODUÇĀO}

A assistência de enfermagem relacionada às necessidades psicossociais dos pacientes parece não estar sendo devidamente valorizada no åmbito das atividades profissionais, uma vez que se percebe uma crescente valorizaçăo das necessidades que se referem à sobrevivência do paciente.

Isso pode ser justificado, em parte, pelo grande avanço tecnológico ocorrido e que tem contribuído para o aprimoramento de técnicas e utilizaçăo de aparelhos e equipamentos altamente sofisticados na área da saúde.

Dessa forma, as alteraçōes orgânicas apresentadas pelo paciente são mais facilmente detectadas e atendidas, contribuindo para que a assistência de enfermagem também seja enfatizada em seus aspectos técnicos ou instrumentais.

Todavia, trabalhos realizados com pacientes cirúrgicos hospitalizados demonstram que eles valorizam, além das atividades técnicas, as atividades de enfermagem da área expressiva ou básica de atuaçäo, isto ê, esperam uma assistência de enfermagem que proporcione segurança emocional e a expressāo de sua individualidade.

MENDES (1986) enfatiza que é necessário considerar que o hospital não ê o "habitat" do eu doente, mas sim, do eu enquanto está doente. $O$ isolamento necessário consequente é uma violência a qualquer pessoa internada, por se tratar de uma circunstáncia que agride o paciente.

ACHCAR (1979) resume a situação de hospitalizado como a perda da cidadania do individuo diante das apreensōes e do sofrimento.

A hospitalizaçăo que, por si, jâ é um fator desencadeante de tensão, pode contribuir ainda mais para o aparecimento de alteraçöes emocionais, quando aliada a um tratamento cirúrgico, que também envolve muitas situaçôes estressantes, como o medo da cirurgia, da anestesia, da dor, de sofrer mutilaçöes, de ser portador de uma doença incurável, além do medo da morte que pode estar presente.

McCLURG (1981) e SANTOS (1972) enfatizam que a falta de orientação e a comunicação falha da equipe de enfermagem acentuam ainda mais as tensōes, inadaptaçōes e insatisfaçöes dos pacientes porque os esclarecimentos fornecidos geralmente são fragmentados, desorganizados e deficientes em prioridades.

Receber informaçöes representa, para o paciente, não só $o$ atendimento às necessidades cognitivas, como o estabelecimento de comunicação interpessoal que pro-

1 - Dissertaçāo de Mestrado apresentada à Escola de Enfermagem da Universidade de Sāo Paulo, sob orientação da Drã Nara Sena de Paula.

2 - Departamento de Enfermagem - CCS/Universidade Estadual de Londrina, Caixa Postal 6001, Londrina - Paraná - Brasil. 
porciona conforto e segurança CHIDA, 1983; ENGSTROM, 1984; KAMIYAMA, 1972; RODRIGUES, 1979; SILVEIRA, 1976: YOKOTA, 1983.

Autores como EPSTEIN (1977), JENNY (1981\} e SOUZA 1976, reconhecem a comunicaçảo enfermeiropaciente, como a essencia da assisténcia de enfermagem e que o estabelecimento de um relacionamento efetivo es o passo inicial para a assistência na área expressiva.

Pelo exposto, percebe-se que a literatura de enfermagem consultada tem enfatizado a importăncia do atendimento das necessidades psicossociais, mas constata-se, também, uma escassez de métodos comprovados de atendimento dessas necessidades.

Com este trabalho de pesquisa, pretendeu-se projetar uma visão das necessidades psicossociais da pessoa hospitalizada para submeter-se a um tratamento cirúrgico do aparelho digestivo, aplicando-se um método sistematizado $\mathrm{e}$ individualizado de assistência de enfermagem (HORTA, 1979), na tentativa de minimizar os efeitos estressantes da hospitalizaçăo, aumentando o bem-estar e o nivel de satisfação do paciente.

\section{2 - OBJETIVOS}

- Verificar o nível de satisfação das necessidades psicossociais de informação, de segurança e de afeto-estima percebidos pelos pacientes submetidos à cirurgia do aparelho digestivo que receberam assistência sistematizada.

- Verificar a relação entre os três niveis de satisfação das necessidades psicossociais: de informação, de segurança e de afeto-estima.

- Verificar se há relação entre o nivel de satisfaçāo das necessidades psicossociais de informação, de segurança e de afeto-estima percebidos pelos pacienes submetidos à cirurgia do aparelho digestivo que receberam assistência sistematizada, com as variáveis sexo, idade e experiência de hospitalização anterior.

\section{3- METODOLOGIA}

Para a assistência sistematizada e individualizada das necessidades psicossociais dos pacientes que fizeram parte dá amostra, foram adotadas duas fases do processo de enfermagem proposto por HORTA (1979); o Histórico e a Evolução de enfermagem.

Utilizou-se também um roteiro com orientaçôes sistematizadas que incluía informaçōes sobre: preparo préoperatório, tratamento cirúrgico, anestesia, centro cirúrgico, condiçōes no pós-operatório e orientaçōes especificas sobre alguns procedimentos que auxiliariam na recuperação pós-operatória.

Para a obtenção dos níveis de satisfação das necessidades de informação, de segurança e de afeto-estima foi elaborado um formulário contendo questões fechadas e abertas (Anexo 1).

Estudos preliminares demonstraram que o tipo de quesitos utilizados nesse instrumento facilitava a com- preensāo por parte dos pacientes.

O trabalho foi realizado em um hospital geral particular da cidade de São Paulo nas unidades de internaçấo médico-cirúrgicas.

A amostra constou de 33 pacientes de ambos os sexos, admitidos para submeter-se à cirurgia do aparelho digestivo no período de janeiro e fevereiro de 1984.

Foram estudadas as seguintes variáveis:

a) Independentes: sexu, idade e experiência de hospitalizaçóes anteriores.

b) Dependentes: nivel de satisfação da necessidade de informaçấ: da necessidade de segurança e da necessidade de afeto-estima.

O nivel de satisfação da necessidade de informação t dado por:

$$
1=\frac{\sum{ }_{i} P_{i}}{M_{i}}=100
$$

em que: $P_{\mathrm{i}}$ es a pontuaçāo de cada indicador e: $\mathrm{M}_{\mathrm{i}}$ é o valor máximo da variável.

O nivel de satisfação da nacessidade de segurança é obtido por:

$$
S=\frac{\sum j^{P_{j}}}{M_{S}}=100
$$

em que: $P_{j}$ é a pontuação de cada indicador e: $\mathrm{M}_{\mathrm{s}}$ é o valor máximo da variável.

O nível de satisfaçăo da necessidade de afeto-estima é dado por:

$$
A=\frac{\sum_{k} P_{k}}{M_{a}}=100
$$

em que: $\mathrm{P}_{\mathrm{k}}$ é a pontuaçäo de cada indicador $\mathrm{e}$; $\mathrm{M}_{\mathrm{a}}$ é o valor máximo da variável.

A coleta de dados foi realizada mediante o acompanhamento sistematizado diário dos 33 pacientes, desde as primeiras 24 horas após a admissão no hospital, até o $4^{\text {ㅁ }}$ dia do pós-operatório ou até a alta hospitalar, quando anterior a esse dia. Nessa ocasiāo, aplicou-se o formulário para a avaliação da assistência implementada.

Para o tratamento dos dados optou-se pela adoção de métodos estatísticos não-paramétricos.

$\mathrm{Na}$ análise de amostras relacionadas utilizou-se a prova de Quade e para as amostras nâo relacionadas utilizaram-se as provas de Mann-Witney e de Kruskal-Wallis.

Em quaisquer situaçőes, o nivel de confiança estabelecido foi de $95 \%$, correspondendo ao erro $\alpha$ de 0,05 .

\section{4 - RESULTADOS}

A tabela 1 registra os pacientes submetidos à cirurgia do aparelho digestivo que receberam assistência siste- 
matizada e os respectivos níveis de satisfaçâo das necessidades de informaçăo, de segurança e de afeto-estima.

TABELA 1 - PACIENTES AMOSTRADOS E SEUS NVVEIS DE SA TISFACRO DAS MECESSIDADES PSICOSSOCIAIS.

\begin{tabular}{|c|c|c|c|c|}
\hline \multirow{2}{*}{ Paclente } & \multicolumn{3}{|c|}{ Nivel do Sotisfaglo da Noceseidade } & \multirow{2}{*}{$\begin{array}{l}\text { Diferençe } \\
\text { Masxime }\end{array}$} \\
\hline & Informaşo & Soguranga & Afeta-Eetima & \\
\hline 1 & 78 & 92 & 75 & 17 \\
\hline 2 & 100 & 83 & 100 & 17 \\
\hline 3 & 96 & 100 & 100 & 4 \\
\hline 4 & 82 & 100 & 88 & 18 \\
\hline 5 & 89 & 100 & 100 & 11 \\
\hline 6 & 93 & 100 & 100 & 7 \\
\hline 7 & 79 & 92 & 100 & 21 \\
\hline 8 & 82 & 100 & 100 & 18 \\
\hline 9 & 43 & 100 & 100 & 57 \\
\hline 10 & 68 & 87 & 100 & 33 \\
\hline 11 & 93 & 92 & 75 & 18 \\
\hline 12 & 86 & 100 & 100 & 4 \\
\hline 13 & 71 & 83 & 88 & 17 \\
\hline 14 & 82 & 100 & 89 & 18 \\
\hline 15 & 89 & 100 & 100 & 11 \\
\hline 18 & 61 & 92 & 100 & 39 \\
\hline 17 & 68 & 83 & 100 & 32 \\
\hline 18 & 36 & 100 & 50 & 64 \\
\hline 19 & 79 & 100 & 88 & 21 \\
\hline 20 & 68 & 100 & 75 & 32 \\
\hline 21 & 96 & 85 & 75 & 21 \\
\hline 22 & 88 & 100 & 75 & 25 \\
\hline 23 & 75 & 100 & 75 & 25 \\
\hline 24 & 71 & 75 & 100 & 29 \\
\hline 25 & 88 & 100 & 60 & 50 \\
\hline 26 & 96 & 100 & 100 & 4 \\
\hline 27 & 8 & 100 & 75 & 25 \\
\hline 28 & 61 & 100 & 100 & 39 \\
\hline 29 & 75 & 100 & 100 & 25 \\
\hline 30 & 79 & 100 & 75 & 25 \\
\hline 31 & 68 & 100 & 100 & 32 \\
\hline 32 & 93 & 100 & 100 & 7 \\
\hline 33 & 93 & 100 & 100 & 7 \\
\hline
\end{tabular}

Os resultados demonstram que os niveis de satisfaçăo das necessidades psicossociais de informaçāo, de segurança e de afeto-estima obtidos, apresentaram uma média amostral respectiva de aproximadamente 79,95 e 89 pontos.

Aplicando-se a prova de Quade, verificou-se que o nivel de satisfaçāo da necessidade de informaçäo é menor que o de segurança e de afeto-estima e que não existe diferença' significativa entre os niveis de satisfaçäo nas duas últimas necessidades.

Utilizando-se a prova de Mann-Whitney, verificou-se que o nivel de satisfação da necessidade de informação é o mesmo para os dois sexos.

Empregando-se a prova de Kruskal-Wallis, percebeuse que o nivel de satisfação da necessidade de informação é o.mesmo tanto em pacientes de diferentes grupos etários, como para pacientes com experiência ou nāo de hospitalização anterior.

Os resultados referentes ao nível de satisfaçäo da necessidade de segurança mostrou-se maior entre os pacientes do sexo feminino, nāo havendo diferenças entre os diversos grupos etários e entre pacientes com ou sem experiência anterior de hospitalizaçāo.

\section{5 - DISCUSSĀO}

Os resultados obtidos demonstram que o método adotado para o atendimento das necessidades psicossociais de informação, de segurança e de afeto-estima dos pacientes submetidos a cirurgia do aparelho digestivo foi satisfatório.

Esperava-se que o nlvel de satisfação da necessidade de informaçâo obtivesse maior pontuação, uma vez que todas as informaçōes solicitadas e as dúvidas apresentadas pelo paciente foram amplamente debatidas individualmente, alérn do fornecimento das demais informaçōes que faziam parte da orientaçāo planejada.

Autores como CASTELLANOS (1976), ROBINSON (1974), WOLFER \& DAVIS (1970), afirmam que o período pré-operatório e principalmente as 24 horas que antecedem a cirurgia, é uma fase em que todos os temores do paciente estāo exacerbados, interferindo na capacidade de compreensäo de um indivíduo.

Diante dessa realidade, pode-se supor que, talvez, o momento mais oportuno e propício para iniciar a orientação pré-operatória seja aquele em que a cirurgia é programada.

De acordo com TAYLOR (1965), pacientes que vivenciam sentimentos de crise pessoal no momento da hospitalizaçāo, poderāo estar incapacitados para relembrar e avaliar as informaçöes recebidas.

Embora a anślise quantitativa não tenha revelado nenhuma relaçāo entre as variáveis independentes e o nivel de satisfação da necessidade de informaçăo, a análise isolada de cada caso permite inferir que essa necessidade só $e$ adequadamente satisfeita, quando se considera a unicidade de cada pessoa.

A assistencia sistematizada individualizada para o atendimento das necessidades psicossociais satisfez, em maior grau, a necessidade de segurança.

Esse método de assistência proporcionou o desenvolvimento das atividades de enfermagem relacionadas à área expressiva de atuação, principalmente pela valorização da interação enfermeiro-paciente.

O contato interpessoal interessado é insubstituivel na enfermagem, quando confrontado com qualquer outro recurso ou instrumental (DANIEL, 1983).

Como afirma STEFANELLI \{1981\}, năo existem fórmulas mágicas para enfrentar os inúmeros e complexos fatores que norteiam o relacionamento enfermeiro-paciente, uma vez que cada situaçăo interpessoal é única.

O fato de se criar um espaço onde uma pessoa pode dizer o que sente sem achar que está sendo cerceada ou inoportuna, representa o verdadeiro apoio, porque quase sempre é justamente isso que a alivia (MALDONADO, 1983).

A terceira necessidade psicossocial estudada referiu-se a necessidade de afeto-estima que obteve um nivel de satisfaçăo muito significativo, comprovando a validade da metodologia adotada.

Conforme KRON \{1978), na prática atual, o enfermeiro 
se vê cada vez mais preocupado com deveres técnicos, de supervisăo e administraçāo e sendo cada vez menos capaz de estar ao lado de seus pacientes. Esse conflito entre - que o enfermeiro aprendeu e o que ele agora realmente faz é o principal problema da enfermagem atual.

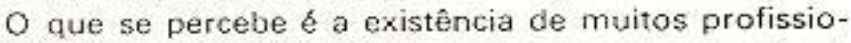
nais eficientes no desempenho de suas atividades técnicas, mas que demonstram grande indiferença quanto ao valor das relaçōes interpessoais como principal recurso no atendimento das necessidades psicossociais dos pacientes.

Tendo em vista as variáveis bio-sociais, pode-se inferir que as necessidades sentidas pelos pacientes sāo basicamente as mesmas para todos, com diferenças individuais nas manifestaçöes e atendimento, corroborando as afirmaçöes de KAMIYAMA \& NAKAZAWA (1976).

Assistir uma pessoa em suas necessidades emocionais nāo é achar a palavra certa para dizer, tentando afastar a emoção e resolver o problema instantaneamente. É permitir que ansiedades, medos e afliçōs sejam expressados, divididos e clareados (WOODHAMS, 1984).

Para ORLANDO (1978), a assistência de enfermagem sistematizada deliberativa dá subsídios para o caráter único de qualquer situaçäo enfermeiro-paciente, fornecendo elementos de contínua refilexão, o que pode propiciar uma prática de enfermagem mais globalizada e eficaz.

Diante do exposto, percebe-se que a assistência de enfermagem a nivel psicossocial ê viável, desde que o enfermeiro se disponha a conferir um lugar de destaque a essa necessidade, preocupando-se em assistir o paciente como uma pessoa, centro de interesse e de atenção de todos os integrantes da equipe de saúde.

Enquanto se pensar na assistência de enfermagem apenas em termos de "ter o trabalho feito", sem a valorizaçăo do desenvolvimento de um relacionamento interessado com o bem-estar das pessoas, estaremos estimulando o distanciamento e a impessoalidade nesse contato.

Dependendo do tipo de interação estabelecida com o enfermeiro, o paciente conseguirá dividir seus senti- mentos, medos e preocupaçōes livremente, permitindo com isso, serem ajudados de acordo com suas necessidades individuais.

Vale enfatizar que a enfermagem nāo é apenas uma profissão técnica que manipula conhecimentos ou tecnologias. É principalmente um trabalho de promoção do ser humano onde sua liberdade, unicidade e dignidade devem ser sempre respeitadas (MENDES, 1976).

\section{6 - CONCLUSŌES}

- Os niveis de satisfação das necessidades psicossociais de informação, de segurança e de afeto-estima, percebidos pelos pacientes submetidos à cirurgia do aparetho digestivo que receberam assistência sistematizada apresentaram uma média amostral de respectivamente, 79,95 e 89 pontos numa escala de 0 a 100.

- O nível de satisfaçāo da necessidade de informaçắo foi menor que os niveis de satisfação tanto da necessidade de segurança, como da necessidade de afeto-estima.

- Näo houve diferença significativa entre os níveis de satisfação das necessidades de segurança e de afeto-estima.

- Os nlveis de satisfação das necessidades de informaçáo e de afeto-estima foram os mesmos para os:

- dois sexos;

- diferentes grupos etários:

- diferentes grupos com experiência de hospitalizaçōes anteriores.

- O nivel de satisfação da necessidade de segurança, percebido pelos pacientes submetidos a cirurgia do aparelho digestivo que receberam assistência sistematizada foi:

a) o mesmo nos:

- diversos grupos etários

b) maior entre os pacientes do sexo feminino.

c) menor entre os pacientes com nenhuma experiência de hospitalização anterior do que entre os pacientes com:

- uma experiência de hospitalização anterior para tratamento clínico ou cirúrgico; $e$

. duas ou mais experiências de hospitalizaçōes anteriores para tratamento clínico ou cirúrgico.

TAKAHASHI, O.C. Psychosocial needs by patients who have undergone surgery on the digestive tract assisted by

systematic and personal nursing care. Semina: Ci. Biol/Saúde, Londrina, v. 13, n. 2, p. $69-74$, june 1992.

\section{ABSTRACT}

This study had as its main purpose to check the level of meeting the psychosocial needs on information, securily, love-esteem noticed by patients who have undergone surgery on the digestive tratc, assisted by systematic and personal nursing care. This assistance was increased from the first 24 hours after the patient admission to the hospital, in the pre-operative period, up to the fourth day post-operative, or before that date, when the patient was discharged. The developed methodology favoured the establishement of a positive nurse-pationt interaction, leading the patient to have the opportunity of clearing their doubts and receiving information about probable happenings which he would undergo during his stay at the hospital for surgery. By the analysis of the results, it was verified that the levels of meeting the needs on information, security and love-esteem presented a sample average of 79,95 and 89 points respectively, ina a 0 -100 scale, representing acceptable levels in the context of this work.

KEY-WORDS: Nursing care; Psychosocial needs; Prל-operative; POs-operative; Assessment; Nursing care systematization; Information; Surgery; Nursing process; Process; Evaluation.

Semina Ci. Biol,/Saúde, Londrina, v. 13, n. 2, p. 69 - 74, jun. 1992 


\section{ANEXO 1}

\section{Formulário de avaliaçāo}

\section{IDENTIFICAÇĀO}

Q: ... L L: ... Data: ... No do formulário: .... Idade: . . . . Sexo: . . . . Religiâo: . . . . . Escolaridade: ......... Ocupaçāo: . . . . . . . . Data da admissão: ..... Data da cirurgia: . . . . . . Cirurgia realizada: .................... Experiências anteriores de hospitalizaçāo e cirurgia: ....

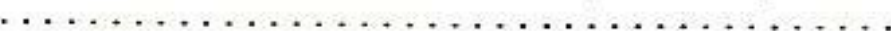

1. Em que grau o esclarecimento de suas dúvidas contribuiu para o (a) Sr (a) enfrentar esta hospitalização para submeter-se à cirurgia?

muito grande

grande

regular

pequeno

nenhum

$\begin{array}{ll}1 & 3 \\ 1 & 1 \\ 1 & 1 \\ 1 & 1 \\ 1 & 1\end{array}$

2. Em que grau as informaçōes apresentadas sobre o tratamento cirürgico, contribuíram para o (a) $\mathrm{Sr}$ (a) enfrentar esta hospitalizaçāo para cirurgia? muito grande

grande

regular

pequeno

nenhum

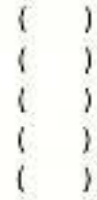

3. Em que grau as informaçōes apresentadas sobre a anestesia contribuíram para o (a) $\mathrm{Sr}$ (a) enfrentar esta hospitalização para cirurgia?

muito grande

grande

regular

pequeno

nenhum

$\begin{array}{ll}1 & 1 \\ 1 & 1 \\ 1 & 1 \\ 1 & 1\end{array}$

4. Em que grau as informaçōes apresentadas sobre o preparo pré-operatório necessário, contribuíram para - (a) Sr (a) enfrentar esta hospitalizaçāo para cirurgia?

muito grande

grande

regular

pequeno

nenhum

$\begin{array}{ll}1 & 1 \\ 1 & 1 \\ 1 & 1 \\ 1 & 1\end{array}$

5. Em que grau as informaçōes apresentadas sobre o centro cirúrgico, contribuíram para o (a) $\mathrm{Sr}$ (a) enfrentar esta hospitalização para cirugia?

muito grande

grande

regular

pequeno nenhum

13

6. Em que grau as informaçōes apresentadas sobre as suas prováveis condiçōes no pós-operatório, contribuíram para o (a) Sr (a) enfrentar esta hospitalizaçāo para cirurgia? muito grande

grande

regular

pequeno

nenhum

$\begin{array}{ll}1 & 1 \\ 1 & 1 \\ 1 & 1 \\ 1 & 1\end{array}$

7. Em que grau as orientaçōes sobre respiração profunda, tosse, mudança de posiçāo, exercícios com os pés e pernas e deambulação no pós-operatório, contribuiram para o seu bem-estar? muito grande grande regular pequeno nenhum

$\begin{array}{ll}1 & 1 \\ 1 & 1 \\ 1 & 1 \\ 1 & 1\end{array}$

8. Qual a informação que o (a) $\mathrm{Sr}$ (a) considerou mais importante ter recebido para enfrentar esta hospitalização para cirurgia? . . . . . . . . . . . . .

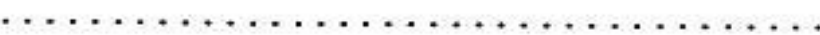

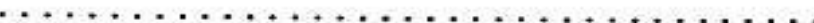
$\ldots \ldots \ldots \ldots \ldots \ldots \ldots \ldots \ldots \ldots \ldots \ldots \ldots \ldots \ldots$

9. O atendimento proporcionado pela pesquisadora contribuiu para eliminar o seu receio (temor) desta hospitalização? completamente em grande parte regularmente em pequena parte em nada

$\begin{array}{ll}1 & 1 \\ 1 & 1 \\ 1 & 1 \\ 1 & 1\end{array}$

10. O relacionamento enfermeiro-paciente estabelecido, contribuiu para o (a) Sr (a) sentir-se tranquilo durante esta hospitalização? completamente em grande parte

$\begin{array}{ll}1 & 1 \\ 1 & 1 \\ 1 & 1 \\ 1 & 1\end{array}$

em pequena parte em nada

11. A confiança que a equipe de entermagem the inspirou foi:

absoluta

grande

regular

pouca

nenhuma

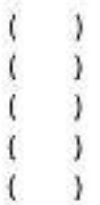


12. O que o $(a\rangle \operatorname{Sr}\langle a\}$ achou mais importante receber da equipe de enfermagem para sentir-se em segurança?



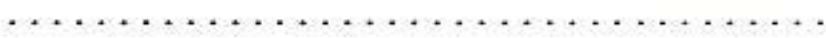
$\ldots \ldots \ldots \ldots \ldots \ldots \ldots \ldots \ldots \ldots \ldots \ldots \ldots \ldots \ldots \ldots \ldots \ldots \ldots$

13. A atenção (apoio) recebida durante a hospitalizaçăo foi: muito grande grande regular pouca nenhuma
14. O respeito e a consideração recebica durante a hospitalização foi:

muito grande

grande

regular

pouco

nenhum

15. Em que ocasiäo o (a) $\mathrm{Sr}$ (a) achou mais importante receber manifestaçōes de afeto-estima lapoiol? dos familiares .....................

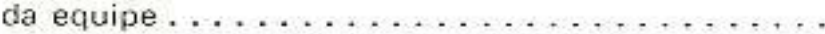

\section{REFERÊNCIAS BIBLIOGRÁFICAS}

ACHCAR, M.C.H. Humanização do hospital. Ent. Atual, Rio de Janeiro, v. 1, n. 5, P. 16-22, jun. 1979.

CAstellanos, B.E.P. Pessoal de centro cirúrgico. Rev. Paul. Hosp., Sāo Paulo, v. 24, n. 6, p. 247-249, jun. 1976.

CHIDA, A.M. informaçós prioritárias ao doente contagioso: estudo comparativo entre pacientes e enfermeiras. Săo Paulo, 1983. 112p. Dissertaçăo (Mestrado) - Escola de Enfermagem da USP.

DANIEL, L.F. Attudes intenpessoais em entermagem, Săo Paulo: EPU, 1983. 176P

ENGSTRŌM, B. The patients'need for information during hospital stay. Int. J. Nurs. Stud., Oxford, v. 21, n. 2, p. 113-130, 1984.

EPSTEIN, C. Interaçăo efetiva na entermagem. Săo Paulo: EPU, 1977. 173p.

HORTA, W.A. Enfermagem: teoria das necessidades humanas básicas. Enf. Novas Dimens, Sāo Paulo, v. 5, n. 3, p. 133-136, maio/jun. 1979.

JENNY, J. Why don't nurses to patient centered nursing? Can. Nurse, Montreal, v. 77 , n. 11, p. 22-25, dec. 1981.

KAMIYAMA, Y. Odoonto hospitalizaolo e sua percepcáo quanto a prioridade de seus problemás. Săo Paulo, 1972. 111p. Tese (Doutorado) - Escola de Enfermagem da USP.

KAMIYAMA, Y,; NAKAZAWA, C.K. Prolemas sentidos por pacientes de um hospital particular de São Paulo. Enf. Novas Dimens, Săo Paulo, v. 1, n. 6, p. 354-359, jan./fev. 1976.

KRON, T. Manual de enfermagem, 4-ed. Rio de Janeiro: Interamericana, 1978. 251p.

McCLURG, E, Developing an effective patient teaching program. AOFN J., Denver, v. 34, n. 3, p. 474-486, sept. 1981.

MALDONADO, M.T. Comunicaço entre aais e filhos: a linguagem do sentir. Petrópolis: Vozes, 1983. 168p.

MENDES, I.A.C. Interaçăo verbal em sifuaçбos de enfcrmagem hospitakr: enfoque humanístico. Ribeirão Preto, 1986. 175p. Tese (Doutorado) - Escola de Enfermagem de Ribeiräo Preto da USP.
ORLANDO, I.J. Orelacionamenio dinấmico entermeiro-paciente. Săo Paulo: EPU, 1978. 110p.

ROBINSON, L.A. Patient's information base: a key to care. Can Nurse, Montreal, v. 70 , n. 12, p. 34-36, dec. 1974.

RODRIGUES, A.I. O paciente no sistema centro cirúrzico; um estudo sobre percepçăo e opiniōes de pacientes em relaçăo ao perfodo transoperatório. Sāo Paulo, 1979. Dissertação (Mestrado) - Escola de Enfermagem da USP.

SANTOS, A.L.V. dos. Contribuiçāo ao estudo da comunicaçāo paciente-equipe de enfermagem, relativa à medicacăo. Säo Paulo, 1972. 49p. Tese (Doutorado) - Escola de Enfermagem da USP.

SILVEIRA, G.C.X. Importáncia das intomacóes ao paciente rocdm-hospitalizado. Salvador, 1976. 101p. Tese (Livre Docência) - Escola de Enfermagem da UFBA.

SOUZA, M.F. Efeito da interaḉco enfemeira-paciente como méíodo de atendimento a necessidade de seguranca do paciente cirturgica. Porto Alegre, 1976. 43p. Tese (Livre Docência) Escola de Enfermagem da UFRS.

STEFANELLI, M.C. Importância do processo de comunicaçāo na assisténcia de enfermagem. Rev, Esc. Ení. USP, Săo Paulo, v. 15 , n. 3, p. 239-245, dez. 1981.

TAYLOR, C.D. The hospital patient's social dilemma. Amer. $t$. Nurs. New York, v. 65, n. 10, p. 96-99, Oct. 1965.

WOLFER, J.A.; DAVIS, C.E. Assessment of surgical patients preoperative emotional conditions and postoperative welfare. Nurs, Res., New York, v. 19, n. 5, p. 402-414, septJoct. 1970 .

WOODHAMS, P. The nurse and the psichologist. Nurs rimes, London, v. 80, n. 1, p. 22-24, jan. 1984.

YOKOTA, O. A problemática cancelamento de cirurgia conforms percebida e expressada pelos pacientos: um estudo explorató rio em um hospital - escola do norte do Paraná. Ribeirão Preto, 1983. 117p. Dissertaçăo (Mestrado) - Escola de Enfermagem de Ribeirāo Preto da USP.

Recebido para publicaçăo em 3/10/1991 